\title{
A vision research apparatus for broad luminance range displays
}

\author{
FREDERICK BONATO \\ Saint Peter's College, Jersey City, New Jersey \\ and \\ JOSEPH CATALIOTTI \\ Ramapo College of New Jersey, Mahwah, New Jersey
}

\begin{abstract}
Lightness, the perceived gray shade of a surface, and the perception of self-luminous surfaces-that is, surfaces that appear to glow-have most often been studied with paper displays and computergenerated stimuli presented on CRT monitors. Although both methods are often effective, experiments that require a wide range of luminance values in the same display are often difficult to conduct with paper and computer displays alone. Also, color mode appearance is often an issue when surface color perception is the topic of research; CRT monitors are essentiallylight sources themselves and often appear in the luminous mode of color appearance. Here, we describe an apparatus in which the target is an undetected aperture whose luminance is adjustable. Whereas a typical CRT monitor offers a luminance range of about 100:1, much broader luminance ranges are possible with the described apparatus. Unlike a CRT monitor, the stimulus background will always appear in the surface mode of color perception, and the target(s) can appear as either surface colors or luminous colors. Apparatus modifications are possible, including the addition of a stereoscope or an embedded CRT for creating an adjustable region that is computer controlled.
\end{abstract}

Lightness, or perceived reflectance, is a surface's perceived gray shade that ranges from black to white. As a surface's luminance is raised above white, the surface will eventually come to appear self-luminous, or glowing. These two areas of color science, lightness and luminosity perception, have often been studied using displays made out of paper or painted surfaces of which actual reflectance values vary. Observers typically view paper displays and indicate their perception of a target's gray shade (lightness) or luminous appearance by adjusting a standard, choosing a surface from a chart that most closely matches the target, or making a forcedchoice judgment regarding the target's appearance. A common problem with paper and/or painted displays is

This research was supported by National Science Foundation Grants BCS-0002620 and BCS-0196505. The authors thank Tiziano Agostini, Daniele Zavagno, and an anonymous reviewer for their constructive suggestions regarding this article. The authors acknowledge that the idea of using an aperture as an adjustable target region has occurred to other investigators. While working in Alan Gilchrist's lab, the authors either used or were exposed to equipment designed by Gilchrist in which adjustable luminance target apertures were key features. Keiji Uchikawa and Yasuki Yamauchi have also designed clever aperture devices for color science research. Other investigators may also have designed and used such devices, and if this is the case, we apologize for not mentioning them here. Correspondence concerning this article should be addressed to F. Bonato, Department of Psychology, Saint Peter's College, 2641 Kennedy Boulevard, Jersey City, NJ 07306 (e-mail: fbonato@spc.edu). that it is difficult to control the luminances of the entire visual scene, especially the background. In addition, paper and painted displays often deteriorate over time; the reflectance of surfaces can change due to age, dirt, or illumination. Recently, and for a number of good reasons, the use of computer-driven CRT displays has become common. Here, we describe an apparatus that can be used when paper displays or CRT displays alone are not suitable for a given research project.

\section{Computer Advantages}

Computers have allowed investigators to easily produce lightness stimulus displays by using readily available graphics programs. Stimulus presentation can take place efficiently in terms of both speed and stimulus control. For example, when a within-subjects design is used, computer-driven displays can allow for quick stimulus changes. Computer-driven displays can also help to ensure consistency across conditions in terms of important stimulus features, such as the spatial placement and luminance relationships within the display. The changing of paper displays is usually more cumbersome and requires much more time and effort in order to maintain consistency across conditions and between subjects. This is especially relevant when one uses random assignment. Also, once digitally stored, stimulus patterns remain stable, whereas paper displays often degrade over time, through either wear and tear or natural aging processes that may change the reflective properties of surfaces. 
Lastly, studying lightness with computer images is efficient in that less space is generally required. An entire experiment can often be contained within a small workstation. In short, computers are valuable tools for lightness scientists, and this article should not be interpreted as a wide sweeping criticism of the use of computers to conduct lightness perception research. However, despite the obvious advantages, several disadvantages are associated with the use of computers when certain aspects of lightness and luminosity perception are studied.

\section{Computer Limitations}

Lightness, often referred to as surface lightness, represents a particular mode of color appearance-namely, the surface mode (Katz, 1935). In the surface mode of color appearance, surfaces appear to be opaque and illuminated by either direct or indirect light sources. Although the impression of opaque surfaces can be created on a CRT, the entire CRT screen may sometimes appear as self-luminous, or glowing. In terms of the color phenomenology first described by Katz, the CRT screen sometimes represents the luminous mode of color appearance. The issue of color mode appearance and CRT screens is actually quite complex, the CRT screen sometimes yielding a dual appearance. Images on the CRT can appear as surfaces, and yet, at the same time, the entire CRT can be perceived as luminous. Surfaces that are unambiguously luminous cannot have lightness values associated with them, because they are not perceived to have a reflectance value. Instead, surfaces that appear selfluminous, or glowing, appear as light sources. Therefore, for some types of lightness perception experiments, presenting stimuli on a CRT might not be desirable, given the modal mismatch (surface/luminous) that can present itself with computer-presented displays.

To be fair, some lightness phenomena, such as lightness contrast and assimilation effects, are perceived with paper displays and displays presented on a CRT monitor. Experiments conducted with both types of displays can yield similar patterns of results (Gilchrist et al., 1999). That is to say, the modal mismatch that CRT monitors can create does not seem to be an issue for some types of displays. However, CRT presentation can affect the magnitude of such effects, such as simultaneous lightness contrast (SLC). In SLC, a gray region surrounded by a bright surround appears to be slightly darker than a physically identical (same-luminance) gray region surrounded by a darker surround. Agostini and Bruno (1996) have shown that the SLC effect is significantly enhanced when the display is presented on a CRT monitor.

Computer displays may raise problems when the hypothesis being tested involves the measurement of when one mode of color appearance crosses over to another. For example, measuring the luminosity threshold (Bonato \& Gilchrist, 1994, 1999), the luminance level at which a surface begins to appear self-luminous, was not possible using computer-generated stimuli alone. In these types of experiments, what is essentially being tested is the lu- minance level at which the surface color mode gives way to the luminous mode. Consider asking a subject to adjust the luminance of a target region on a CRT until it reaches the luminosity threshold. The task becomes complicated in that, under some conditions, the entire CRT screen may already appear to be self-luminous.

Another problem to consider is luminance range. Consider that luminous surfaces are those that appear brighter than a white surface could possibly be at a given level of illumination (Hering, 1874/1964). In short, in order to make a surface appear self-luminous - that is, brighter than white-its luminance under normal circumstances must be above that of a white surface in the same illumination framework. The reflectance range of white to black surfaces in the same illumination is about $30: 1$; a good white surface will reflect about $90 \%$ of the illumination striking it, as opposed to a black surface, which will reflect about $3 \%$ of its illuminant. In some studies, the mean luminosity threshold was reached when a target's luminance was about five times higher than that of a white surface in the scene (Bonato \& Gilchrist, 1999). Because the luminances of white surfaces in those experiments were 30 times higher than the luminances of black surfaces in the same illumination, another way of expressing the luminosity threshold is to say that the threshold was reached when the target's luminance was 150 times higher than that of a black surface in the scene. However, some subjects, when adjusting a target to reach the luminosity threshold, require a much higher luminance range, often several hundred to one (Bonato \& Cataliotti, 2000). Typical CRT monitors have a luminance range of about 100:1, and even much more expensive models with expanded range capabilities do not offer the luminance range required for many luminosity threshold experiments.

To be fair, we note that Zavagno and Caputo (2001) have used CRT monitors to measure the luminosity threshold. However, their displays were very different from those used by Bonato and colleagues (Bonato \& Cataliotti, 2000; Bonato \& Gilchrist, 1994, 1999), in that wide luminance ranges were not required. In their experiments, a homogeneous square target region was made to appear self-luminous simply by flanking it with four regions containing luminance gradients. The lightest areas of the gradients were adjacent to the central target region. The resulting cross-pattern created the impression that the center target was self-luminous, an impression that increased as the steepness of the luminance gradients surrounding the target was increased. The luminous appearance of the central target region, or glare effect, can even be experienced with a paper display having a luminance range of 30:1. Therefore, although wide luminance ranges are sometimes required for luminosity perception research, for some experiments, other methods are possible.

Another problem for lightness researchers to consider is that CRT monitors may need to be calibrated. There are at least two types of calibration problems. When a 
target region's luminance is changed on an uncalibrated monitor, the target's chromaticity may also change. The output of the three CRT "guns" that produce the CRT image may not fire proportionally at all output levels. Therefore, what appears to be a completely achromatic gray at $10 \mathrm{~cd} / \mathrm{m}^{2}$ may appear to be chromatically tinted at $100 \mathrm{~cd} / \mathrm{m}^{2}$ - an undesirable arrangement for an experiment specifically designed to test lightness. Also, as target luminance is changed on a CRT monitor, the luminance of other regions on the monitor may also change. For example, as the luminance of a target region is raised, the luminance of surrounding regions may be lowered. In short, regions of a CRT display typically exert lateral inhibitory effects on each other.

The apparatus described below, which can be constructed of readily available, inexpensive materials, has some advantages over both traditional paper displays and computer displays and addresses many of the problems previously discussed. The apparatus can be easily modified to interface with a CRT monitor and offers the opportunity to present high luminance range displays stereoscopically.

\section{Apparatus}

A schematic drawing of the apparatus is shown in Figure 1. It consists of an elongated chamber $38 \mathrm{~cm}$ wide, $31 \mathrm{~cm}$ high, and $74 \mathrm{~cm}$ long. Viewing takes place through a $2.5 \times 13 \mathrm{~cm}$ slot cut out from one end of the chamber. When looking through the slot, the observer sees the stimulus display located on the far wall of the viewing chamber, $41 \mathrm{~cm}$ from the viewing slot. For illustration purposes, a sample stimulus display is shown in Figure 2. We have used both hand-cut paper displays and displays printed with high-resolution printers and a graphics program, such as Canvas or Photoshop. The far wall of the viewing chamber is actually a panel that can slide in two
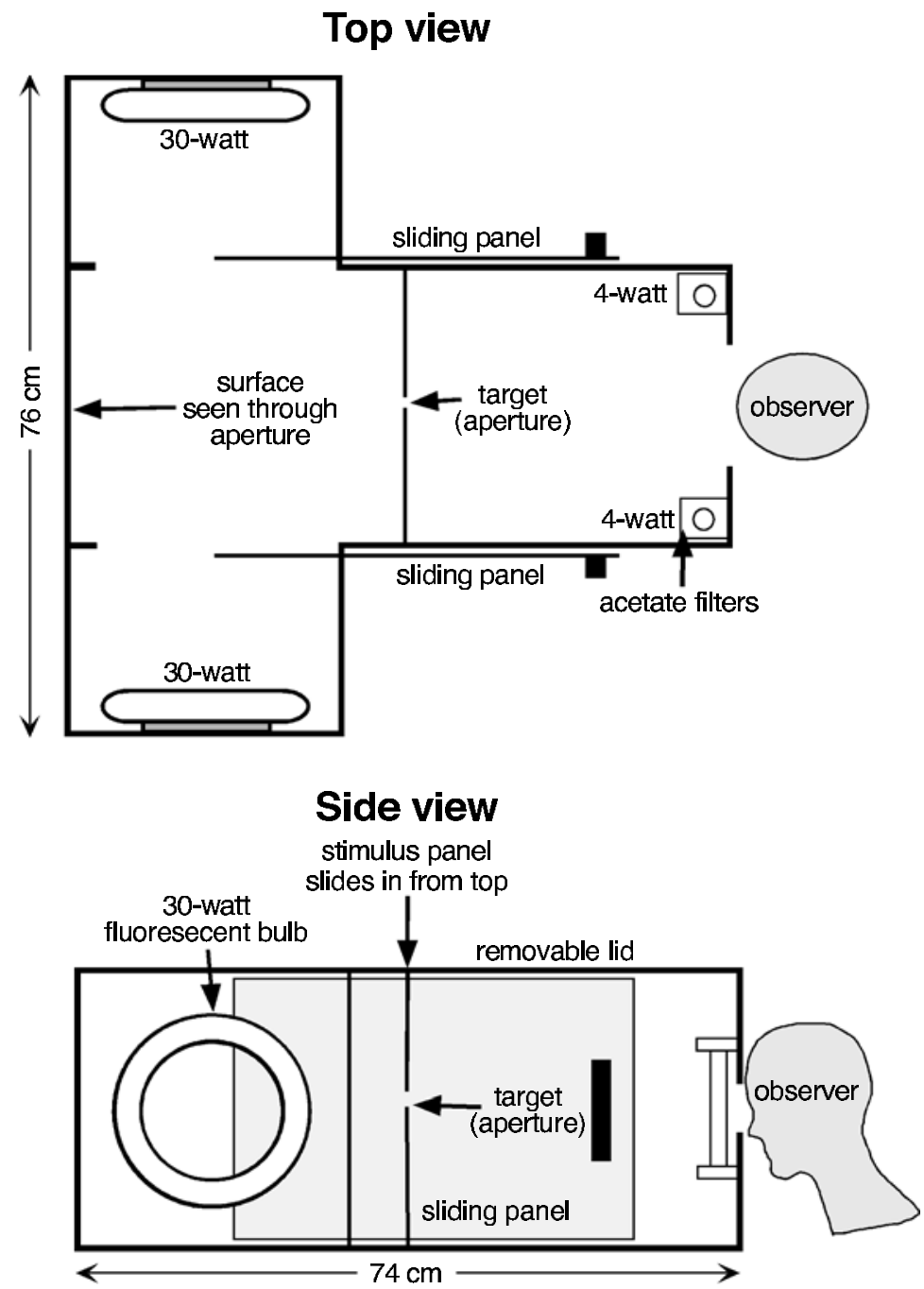

Figure 1. A schematic drawing of the apparatus. 


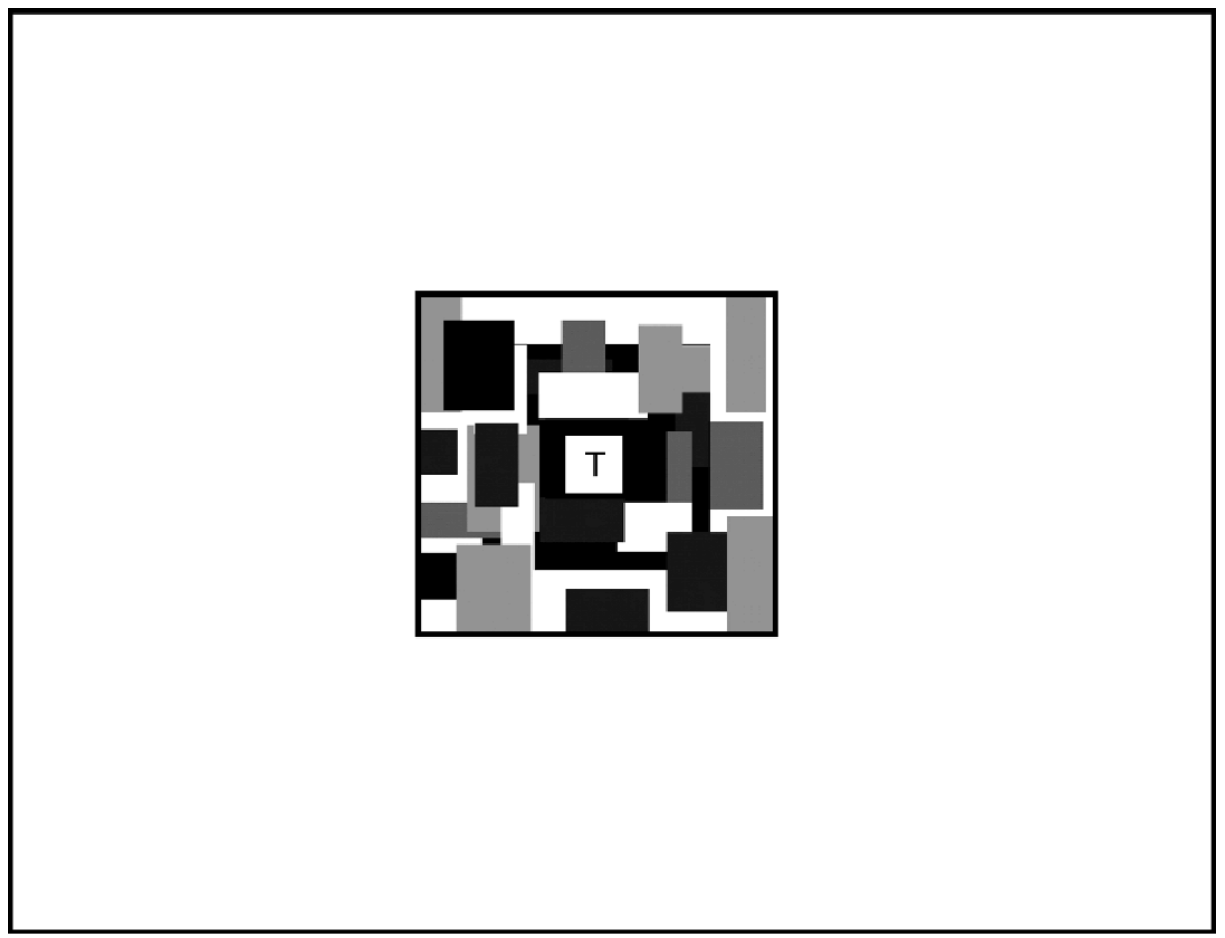

Figure 2. A sample stimulus pattern. Although it can appear as either a flat opaque surface or a luminous surface, the target $(T)$ is actually an aperture whose luminance is adjustable by the observer.

vertical tracks. The stimuli can be changed by removing the lid of the apparatus and sliding a new stimulus panel into place.

Although the target, whose luminance is adjustable, appears to be coplanar with the rear wall of the viewing chamber, it is actually a carefully cut aperture that opens to a separately illuminated chamber located behind the viewing chamber. The surface seen through the aperture is actually a piece of paper mounted on the interior rear wall of the apparatus. Although we have run hundreds of subjects in various experiments, no one, to date, has ever correctly identified the target as an aperture; instead, the target region consistently appears as a surface.

When looking at the stimulus display, the observer can adjust the brightness of the target by grasping two handles, one positioned on each side of the apparatus. Pushing the handles forward causes the target to become dimmer, and pulling the handles causes the target to become brighter. Each handle is attached to a sliding panel that rides in aluminum tracks mounted to the sides of the chamber. When pushed forward, the panels move in tandem to close off two small chambers attached to opposite sides of the apparatus's rear chamber. The interior of each small chamber is painted matte white, to facilitate light diffusion. Each of these small chambers contains a 25-cm-diameter 30-W circular fluorescent bulb. When the handles are pulled, light is allowed to spill into the rear chamber, raising the luminance of the rear wall, which is observed though the aperture that serves as the target. Like the small chambers that contain the fluorescent bulbs, the rear chamber's interior is also painted matte white. This results in an abundance of reflected light within the rear chamber, which helps maintain a uniform luminance of the rear wall seen through the aperture. We have successfully used target apertures having uniform luminance that subtended up to $3^{\circ}$ of visual angle. Although we have not done so, using larger apertures while maintaining target homogeneity should be possible within limits. Using a larger apparatus, with a larger rear chamber, would allow for experiments in which even larger apertures are employed.

Although the luminance range capability of the apparatus will depend on the type of display used, we have achieved a luminance range of over 700:1. The luminance range of the apparatus can be modified by changing the physical reflectance of the surface seen through the aperture, changing the strength of the bulbs in the rear chamber, or both.

The near viewing chamber is illuminated by two 4-W fluorescent bulbs mounted to the viewing chamber's interior, one on each side of the viewing slot, hidden from direct view by the observer. The level of illumination within the near viewing chamber can be controlled and chromatic differences between the target aperture and the near viewing chamber's interior eliminated by placing acetate filters in front of the bulbs. In experiments 
conducted with the apparatus (Bonato \& Cataliotti, 2000, 2002), chromatic differences were eliminated by placing a white background in the viewing chamber and adjusting the luminance of the target aperture until it matched the appearance of the white background as closely as possible. With this technique, chromatic differences between the target and the background become most apparent. Depending on the perceived hue of the target, appropriate acetate filters were placed on the $4-\mathrm{W}$ fluorescent bulbs in the viewing chamber. For example, if the target appeared reddish, a reddish filter would be used in an attempt to balance the chromaticity of the background with that of the target. This process was repeated until the target could be made to visually "blend in" with the white background.

\section{Apparatus Modifications}

The apparatus can be custom modified to accommodate specialized needs. For example, we have used it to present stereoscopic stimuli (Bonato \& Cataliotti, 2002).
When the luminosity threshold was measured, targets were presented that appeared to be on a depth plane different from the depth plane constituted by the background. The modification involved the attachment of a mirror stereoscope to the viewing slot (see Figure 3). Two side-by-side images were required for the stimulus display, one side for the left eye and one for the right. Therefore, instead of one aperture, two were needed. Depending on the disparity set for the two side-by-side images fused with the stereoscope, the targets were made to appear in front of, coplanar with, or behind the background. In short, although the target sometimes appeared to be floating in the middle of the viewing chamber or in a space behind the background, its brightness was still adjustable.

Although the authors think displays presented with the apparatus described here might, in some situations, be more suitable than presenting stimuli on a CRT alone, some of the problems that CRT displays potentially present can be avoided by using a hybrid apparatus into which

\section{Stereoscopic modification (top view)}

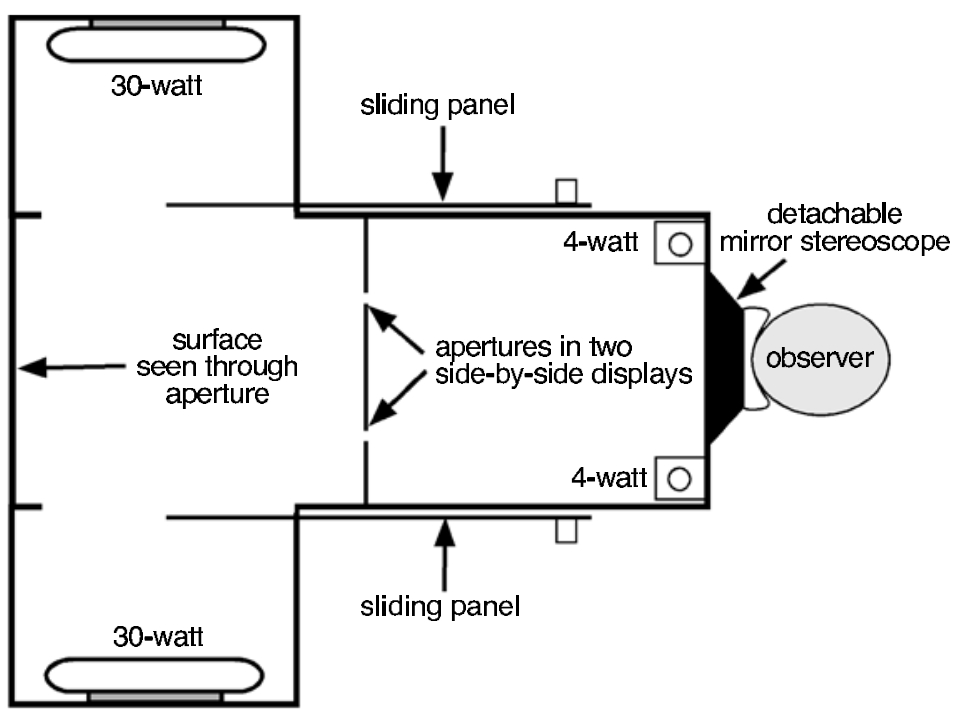

\section{CRT modification (side view)}

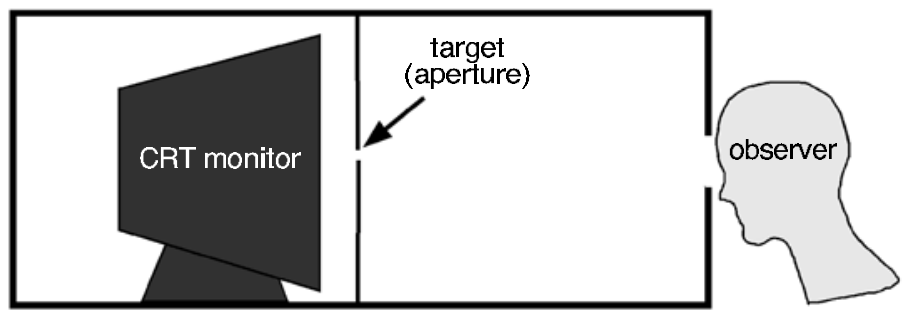

Figure 3. Possible apparatus modifications. In the top diagram, a stereoscope has been attached to the viewing slot, making wide luminance range threedimensional stereoscopic displays possible. In the bottom diagram, a CRT monitor has been positioned behind the target aperture in order to control the target's luminance with a computer. 
a CRT monitor is embedded. ${ }^{1}$ When a broad luminance range is not needed, a CRT monitor can be positioned behind the aperture(s) (see Figure 3 ). Instead of changing target luminance by flooding light into the rear chamber, the target's luminance can be changed with the aid of a computer. Although we have not used this particular type of modification with this apparatus, we have successfully used a CRT monitor in conjunction with an aperture target in another experimental setup (Cataliotti \& Bonato, 2003). Other investigators have also successfully used aperture targets in conjunction with CRT monitors. Yamauchi and Uchikawa (1998, 1999, 2000) have used aperture targets in displays to measure the upper limit luminance for surface color perception under a variety of conditions. The method they used is conceptually similar to those suggested here. For color perception experiments in general, this type of arrangement avoids conflicting color mode appearances that occur with a CRT alone (luminous mode vs. surface mode). The regions that constitute the stimulus background will be experienced as surface colors, and the target will be experienced as either a surface color (if its luminance is low enough) or a luminous color (if its luminance is sufficiently raised).

\section{Summary}

Although paper stimulus displays and those presented on CRTs will undoubtedly continue to be used for lightness and luminosity perception experiments, limitations are associated with both of these methods of stimulus presentation. An apparatus like the one described here may be desirable in certain situations, such as when (1) a broad range of luminance values is needed in a single display, (2) the mode of color appearance of either the target or the context in which it is found is of concern (e.g., surface mode vs. luminous mode), and (3) space limitation is an issue-that is, when the display and the apparatus must be contained within a small work space. Possible modifications include, but are not limited to, (1) a stereo- scope attachment for presenting three-dimensional displays and (2) a CRT for changing target luminance.

\section{REFERENCES}

Agostini, T., \& BRUno, N. (1996) Lightness contrast in CRT and paper-and-illuminant displays. Perception \& Psychophysics, 58, 250258

Bonato, F., \& CAtaliotti,J. (2000). The effects of figure/ground, perceived area, and target saliency on the luminosity threshold. Perception \& Psychophysics, 62, 341-349.

Bonato, F., \& CAtaliotti, J. (2002). Pictorial and stereoscopic grouping effects on the luminosity threshold [Abstract]. Journal of Vision, 2, 550a, http://journalofvision.org/2/7/500/, DOI 10.1167/2.7.550.

Bonato, F., \& Gilchrist, A. L. (1994). The perception of luminosity on different backgrounds and in different illuminations. Perception, 23, 991-1006.

Bonato, F., \& Gilchrist, A. L. (1999). Perceived area and the luminosity threshold. Perception \& Psychophysics. 61, 786-797.

Cataliotti, J., \& Bonato, F. (2003). Spatial and temporal lightness anchoring. Visual Cognition, 10, 621-635.

Gilchrist, A., Kossyfidis, C., Bonato, F., Agostini, T., CatalIOtTi, J., Li, X., SPEhar, B., AnNAN, V., \& ECONOMOU, E. (1999). An anchoring theory of lightness perception. Psychological Review, 106, 795-834.

HERING, E. (1964). Outlines of a theory of the light sense (L. M. Hurvich \& D. Jameson, Trans.). Cambridge, MA: Harvard University Press. (Original work published 1874)

KAtZ, D. (1935). The world of colour. London: Kegan Paul, Trench, Trubner \& Co.

YAMAUCHI, Y., \& UCHIKAWA, K. (1998). Effects of non-uniform illumination on the mode of color appearance [Abstract]. Investigative Ophthalmology \& Visual Science, 39, S897.

YAMAUCHI, Y., \& UCHIKAWA, K. (1999). Luminance limit for surfacecolor mode perception under spatial luminance gradient. Vision, 11, $1-11$.

YAMAUCHI, Y., \& UCHIKAWA, K. (2000). Upper-limit luminance for the surface-color mode appearance. Journal of the Optical Society of America A, 17, 1933-1941.

ZavaGno,D., \& CAPUTO, G. (2001). The glare effect and the perception of luminosity. Perception, 30, 209-222.

\section{NOTE}

1. The use of an embedded CRT monitor may require calibration of the monitor in terms of both chromaticity and luminance homogeneity. 